\title{
Metodología para la Medición de Parámetros de Sobretensiones Transitorias en Redes de Distribución de $11.4 \mathrm{kV}^{*}$
}

\section{Methodology for Measuring Transient Overvoltage Parameters in $11.4 \mathrm{kV}$ Distribution Networks}

\author{
DOI: http://dx.doi.org/10.17981/ingecuc.12.1.2016.06
}

Artículo de investigación científica - Fecha de recepción: 10 de diciembre de 2014 - Fecha de aceptación: 25 de noviembre de 2015

Denis Amanda Acevedo Vásquez

Ingeniera Eléctrica. Universidad Distrital Francisco José de Caldas. Bogotá (Colombia). daacevedov@correo.udistrital.edu.co

\section{Gloria Chacón Pedraza}

Ingeniera Eléctrica. Universidad Distrital Francisco José de Caldas. Bogotá (Colombia). ggchaconp@correo.udistrital.edu.co

\section{Francisco Santamaría Piedrahita}

Doctor en Ingeniería. Universidad Distrital Francisco José de Caldas. Bogotá (Colombia). fsantamariap@udistrital.edu.co

Para citar este artículo / To reference this paper:

D. A. Acevedo Vasquez, G. Chacón Pedraza y F. Santamaría Piedrahita "Metodología para la medición de parámetros de sobretensiones transitorias en redes de distribución de 11.4 Kv", INGE CUC, vol. 12, no. 1, pp. 65-72, 2016. DOI: http://dx.doi.org/10.17981/ingecuc.12.1.2016.06

\begin{abstract}
Resumen-- Debido a que las sobretensiones transitorias son un fenómeno que afecta a los sistemas de potencia, se requieren metodologías que permitan caracterizar este fenómeno a partir de sus parámetros principales (valor pico, duración y frecuencia de oscilación) de una forma práctica y no invasiva. El presente artículo tiene como objetivo presentar la metodología de medición de las sobretensiones en redes de distribución de $11,4 \mathrm{kV}$. Se presentan los conceptos y efectos más importantes acerca del fenómeno en estudio, el desarrollo de la metodología y las conclusiones más importantes de la investigación. La investigación desarrollada tuvo como etapa inicial la elaboración del estado del arte de las sobretensiones en redes de media tensión; posteriormente, se establecieron los parámetros más importantes de las sobretensiones que se buscaban estudiar y registrar; luego se estudiaron diferentes opciones para el diseño del sensor de campo eléctrico; y finalmente, se realizó el diseño detallado, la construcción y las pruebas del prototipo.
\end{abstract}

Palabras claves-- Calidad de potencia eléctrica; campo eléctrico; redes aéreas de media tensión; sistemas de medición; sobretensiones transitorias; medición de fenómenos transitorios.

\begin{abstract}
Given that transient overvoltage is a phenomenon that affects power systems, different methodologies to characterize this phenomenon in a practical and non-invasive way using its main features (peak value, duration and frequency of oscillation) are required. Therefore, the authors of this paper proposed an investigation project: the development of a prototype for the registration of these parameters in $11,4 \mathrm{kV}$ networks using a capacitive electric field sensor, the initial stage for the research carried out for the abovementioned project was the state of the art regarding surges in medium voltage networks; then, the most important parameters of the surge that wanted to be studied and recorded were established. Afterwards, different options for the design of the electric field sensor were analyzed and, then, the detailed design, construction and testing of the prototype was carried out. This paper aims to present the methodology for measuring voltages in distribution networks $11.4 \mathrm{kV}$ developed as part of the investigation previously outlined. Hence, the most important concepts and effects of the phenomenon, the methodology developed and the key findings of the investigation are presented.
\end{abstract}

Keywords-- Power Quality; Electric Field; Aerial Medium Voltage Networks; Measuring Systems; Transient Overvoltage; Measurement of Transient Electrical Phenomena.

\footnotetext{
" Artículo de investigación científica derivado del proyecto de investigación titulado: "Desarrollo de un prototipo para el registro de sobretensiones en redes de 11,4 kV". Financiado por el Centro de Investigaciones y Desarrollo Científico de la Universidad Distrital Francisco José de Caldas. Fecha de inicio: enero de 2013. Fecha de finalización: enero 2014.
} 


\section{METODOLOGÍA PARA LA MEDICIÓN DE PARÁMETROS DE SOBRETENSIONES TRANSITORIAS EN REDES DE DISTRIBUCIÓN DE 11.4 KV}

\section{INTRODUCCIÓN}

El presente artículo presenta parte de los resultados obtenidos durante desarrollo del proyecto de grado titulado: Desarrollo de un prototipo para el registro de sobretensiones transitorias en redes de $11,4 \mathrm{kV}$ [1]. Este proyecto se realizó en las siguientes etapas:

1. Elaboración del estado del arte sobre las sobretensiones en redes de media tensión.

2. Estudio de opciones para el diseño del sensor de campo eléctrico.

3. Diseño detallado del sensor.

4. Diseño detallado de la etapa de adquisición de datos.

5. Construcción y pruebas.

En este artículo los autores presentan la metodología de medición y registro de las sobretensiones que utiliza el prototipo construido. Esta metodología hace parte de los resultados más relevantes de la investigación ya que permite categorizar el fenómeno de acuerdo con sus parámetros más relevantes.

El proyecto de investigación que enmarca los resultados de este artículo toma como punto de partida que una sobretensión se define como cualquier tensión entre fase y tierra o entre fases con un valor pico que exceda el valor pico correspondiente del valor más alto para el equipo [1]. Las sobretensiones se clasifican en dos grandes grupos según su duración: permanentes y transitorias; en esta investigación fueron estudiadas las sobretensiones transitorias. Las sobretensiones transitorias se definen como una sobretensión de corta duración, de unos pocos milisegundos o menos, que puede o no ser oscilatoria y usualmente altamente amortiguada [1].

Las sobretensiones en las redes de media tensión hacen parte de la operación normal del sistema y se presentan, por ejemplo, por maniobras en las subestaciones y redes de media tensión tales como re-cierres de líneas, también se ocasionan debido a fallas fase - tierra en el sistema y debido a descargas atmosféricas directas o inducidas en el sistema. Adicionalmente, pueden ocasionar pérdida del suministro de energía, daños en equipos sensibles, daños en celdas aisladas en SF6, transformadores y líneas de media tensión, lo cual tiene importantes efectos negativos para los elementos que componen el sistema y para las cargas conectadas al mismo. Por lo expuesto anteriormente es importante caracterizar las sobretensiones transitorias de una forma sencilla y económica en comparación con los mecanismos convencionales que existen para realizar esta medición.

Este artículo presenta una metodología económica y de fácil implementación para registrar los principales parámetros de las sobretensiones transitorias en redes aéreas de media tensión. Se exponen cuatro secciones principales. Primero se hace necesario conocer las características más relevantes de las sobretensiones transitorias y, de esta manera, identificar las generalidades del fenómeno. Posteriormente, se exponen los principales efectos de las sobretensiones transitorias en el sistema tomando algunos casos de estudio particulares en las redes de media tensión $\mathrm{y}$, así, poner en evidencia la importancia del estudio de este fenómeno. Adicionalmente, se realiza una breve descripción de los mecanismos más comunes utilizados para la medición de diferentes fenómenos de calidad de potencia, y particularmente, de las sobretensiones transitorias. Por último, se propone una metodología para registrar los parámetros más importantes de las sobretensiones transitorias. Para concluir, se realiza una comparación entre los mecanismos existentes con la metodología propuesta para determinar las ventajas y desventajas de éstas.

\section{Sobretensiones Transitorias}

Las sobretensiones transitorias se pueden clasificar de acuerdo con su forma de onda en las siguientes categorías:

\section{A. Sobretensiones de frente lento}

Es una sobretensión generalmente unidireccional con tiempo de subida T1, $0.1<\mathrm{T} 1<5000 \mu$ s y tiempo de cola T2 $\leq 20 \mathrm{~ms}$. Las causas más comunes de las sobretensiones de frente lento son [2]:

- Energización de redes

- Re-cierre o maniobras en el sistema

- Despeje de fallas monofásicas

- Rechazo de carga

\section{B. Sobretensiones de frente rápido}

Son sobretensiones generalmente unidireccionales de muy corta duración T2, T2 $\leq 300 \mu \mathrm{s}$, tienen una amplitud muy superior al valor nominal del sistema (4 a 6 veces); sus causas más comunes son [3]:

- Descargas atmosféricas conducidas

- Descargas atmosféricas inducidas

- Descargas atmosféricas directas en las redes aéreas

\section{Sobretensiones de frente muy rápido}

El valor pico de estas sobretensiones aumenta rápidamente pues es alcanzado en menos de $1 \mu \mathrm{s}$. La oscilación posterior al frente de onda tiene una frecuencia aproximada máxima de $1 \mathrm{MHz}$ y duración menor a $3 \mathrm{~ms}$. Las causas más comunes de este tipo de sobretensiones son [3]:

- Maniobras en subestaciones de media tensión

- Fallas en subestaciones de media tensión

En la Tabla I se presentan las formas de onda características de cada tipo de sobretensión transitoria junto con sus principales parámetros de acuerdo con lo presentado en la norma IEC 60071-1 [1].

Como ya se estableció, las sobretensiones pueden clasificarse a partir de su forma de onda y este parámetro se relaciona con su causa, por lo cual al 
momento de analizar las sobretensiones, unos de los parámetros más importantes a determinar son:

- Valor pico

- Duración del fenómeno

Estos dos parámetros son el punto de partida que permite categorizar los fenómenos, clasificarlos y estudiar sus efectos.

Tabla I. Principales Características De las Sobretensiones Transitorias.

\begin{tabular}{|c|c|c|c|}
\hline \multirow{2}{*}{$\begin{array}{c}\text { Clase de } \\
\text { sobretensión }\end{array}$} & \multicolumn{3}{|c|}{ Transitoria } \\
\hline & De frente lento & $\begin{array}{l}\text { De frente } \\
\text { rápido }\end{array}$ & $\begin{array}{l}\text { De frente my } \\
\text { rápido }\end{array}$ \\
\hline Forma & $T_{p} \frac{1}{T_{T_{2}}}-$ & $\mathrm{T}_{1} \notin \mathrm{T}_{2}$ & $T_{t} \stackrel{W}{=} T_{t}$ \\
\hline $\begin{array}{l}\text { Gama de forma } \\
\text { (frecuencia, } \\
\text { frente de } \\
\text { subida, } \\
\text { duración) }\end{array}$ & $\begin{array}{c}5000 \mu \mathrm{s}>\mathrm{T}_{\mathrm{p}}> \\
20 \mu \mathrm{s} \\
\mathrm{T}_{2} \leq 20 \mathrm{~ms}\end{array}$ & $\begin{array}{c}20 \mu \mathrm{s}>\mathrm{T}_{1}> \\
0,1 \mu \mathrm{s} \\
\mathrm{T}_{2} \leq 300 \mu \mathrm{s}\end{array}$ & $\begin{array}{c}100 \mathrm{~ns} \geq \mathrm{T}_{\mathrm{f}}>3 \mathrm{~ns} \\
0,3 \mathrm{MHz}<\mathrm{f}_{1}< \\
100 \mathrm{MHz} \\
30 \mathrm{MHz}<\mathrm{f}_{2}<300 \\
\mathrm{MHz} \\
\mathrm{T}_{\mathrm{t}} \leq 3 \mathrm{~ms}\end{array}$ \\
\hline $\begin{array}{c}\text { Forma } \\
\text { normalizada }\end{array}$ & $\begin{array}{l}\mathrm{T}_{\mathrm{p}}=250 \mu \mathrm{s} \\
\mathrm{T}_{\mathrm{p}}=250 \mu \mathrm{s}\end{array}$ & $\begin{array}{l}\mathrm{T}_{1}=1,2 \mu \mathrm{s} \\
\mathrm{T}_{2}=50 \mu \mathrm{s}\end{array}$ & $(*)$ \\
\hline $\begin{array}{l}\text { Ensayo de } \\
\text { tensión } \\
\text { soportada } \\
\text { normalizada }\end{array}$ & $\begin{array}{l}\text { Ensayo de } \\
\text { impulso tipo } \\
\text { maniobra }\end{array}$ & $\begin{array}{c}\text { Ensayo de } \\
\text { impulso tipo } \\
\text { rayo }\end{array}$ & $(*)$ \\
\hline
\end{tabular}

Fuente: [1].

\section{Efectos de las Sobretensiones Transitorias}

Las sobretensiones transitorias tienen importantes efectos negativos sobre los equipos que conforman los sistemas de potencia en alta, media y baja tensión y sobre las cargas de los usuarios residenciales, comerciales e industriales. En esta sección se muestran algunos casos de estudio que ilustran los efectos de este fenómeno sobre las redes.

\section{A. Sobretensiones transitorias en motores}

Cuando se presenta una sobretensión en el sistema de distribución de media tensión, ésta afecta a los equipos conectados a esta red, incluyendo cargas como motores. La magnitud de la sobretensión que afecta al motor depende de diferentes factores, como la topología de la red, las características de los alimentadores, la longitud del circuito alimentador, el tipo de motor y las demás cargas conectadas al circuito y barraje afectados [4]. Aunque estas características son particulares para cada caso, se pueden generalizar como se muestra a continuación:

- Para sistemas con mayores niveles de tensión, el tiempo de ascenso será menor; y para menores niveles de tensión, el tiempo de ascenso será mayor.

- Un mayor número de cargas conectadas al barraje afectado incrementa la magnitud de la sobretensión.
- Una mayor longitud del alimentador del motor incrementa el tiempo de ascenso. Adicionalmente, los cables apantallados, por tener una impedancia menor, hacen que se incremente la magnitud de la sobretensión.

- Los motores de gran potencia verán sobretensiones de menor magnitud y tiempos de ascenso mayores.

\section{B. Sobretensiones ocasionadas por descargas atmosféricas en redes de media tensión}

Las sobretensiones en redes de media tensión ocasionadas por descargas atmosféricas pueden ser de dos tipos:

Descargas atmosféricas directas: Este tipo de descargas atmosféricas ocurre debido a fallas en el sistema de apantallamiento de la línea y ésta es impactada directamente por la descarga atmosférica. Estas sobretensiones tienen valores pico muy altos debido a que las ondas viajeras se propagan en sentidos contrarios a partir del punto de incidencia del rayo y al encontrar un punto de discontinuidad se refleja retornando al punto de incidencia y se superpone con la onda incidente dando como resultado una sobretensión de una magnitud doble [5].

Descargas atmosféricas inducidas: Este tipo de descargas retornan por un canal ionizado cercano a la línea afectada en un radio aproximado de 200 $\mathrm{m}$; la sobretensión es causada por el cambio brusco en el campo electromagnético producido por la corriente de retorno [6]. Este fenómeno ocasiona fallas fase - fase, daños en el aislamiento de las líneas y funcionamiento incorrecto de la red, causando así el $80 \%$ de las fallas en media tensión, y por lo tanto, es importante poder caracterizarlas y así establecer criterios para el diseño de dispositivos de protección (DPS) [7].

\section{Sobretensiones de frente rápido en transformadores de distribución}

Las redes de distribución generalmente utilizan bajos niveles de aislamiento y las cargas sensibles son particularmente susceptibles a las descargas atmosféricas, aspectos negativos de las redes y las cargas conectadas al sistema que puede afectar la confiabilidad de la red y fallas en el suministro, causando pérdidas económicas.

Generalmente, los transformadores de distribución están protegidos contra descargas atmosféricas por descargadores de sobretensiones instalados cerca de los terminales de media tensión del transformador; sin embargo, las fallas de los transformadores pueden ser ocasionadas por descargas atmosféricas originadas en el lado de baja tensión del transformador o por descargas transferidas desde el lado de media tensión del transformador. Estas sobretensiones transferidas se asocian al aumento 
de la tensión en la puesta a tierra del transformador debido a la corriente que fluye a través de los descargadores de sobretensión de media tensión y al acople electromagnético entre los terminales de media y baja tensión [8].

Para los tres casos específicos expuestos anteriormente se pone en evidencia que el estudio y caracterización de las sobretensiones son de gran importancia para mitigar los efectos negativos y diseñar instalaciones confiables y menos vulnerables a los efectos de las sobretensiones transitorias.

\section{Sistemas de Medición de los Fenómenos de Calidad de Potencia}

Dentro de la amplia gama de equipos para la medición de parámetros de calidad de potencia podemos resaltar los siguientes, ya que son los más comunes dentro del mercado:

\section{A. Medidores de calidad de potencia}

Estos medidores funcionan por medio de sondas de tensión y corriente que se conectan de acuerdo con la topología del sistema y las necesidades de medición, por lo general estos equipos pueden registrar los siguientes parámetros:

- Potencia activa

- Potencia aparente

- Potencia reactiva

- Factor de potencia

- Energía (kWh)

- THDV, THDI

- Frecuencia

- Armónicos

Para obtener estos parámetros el equipo registra las ondas completas tanto de tensión como de corriente, realizando así los cálculos de potencia, energía y armónicos. Adicionalmente, se obtienen perfiles de carga y de tensión de acuerdo con las necesidades de los usuarios dentro de unos rangos determinados. Generalmente sólo realizan mediciones hasta 1000 Vrms, trabaja en frecuencias de 50/60 $\mathrm{Hz}$ y tiene un ancho de banda de $1.5 \mathrm{kHz}$ [9].

\section{B. Indicadores de fallas}

Otros equipos conocidos como indicadores de fallas son instalados en las estructuras de las redes de media tensión, principalmente en redes rurales donde se dificulta determinar la ubicación de las fallas y se tienen zonas de difícil acceso. Estos indicadores funcionan con sensores capacitivos y puede detectar fallas de corto circuito, fallas a tierra y fallas fase - fase. Estos dispositivos tienen la posibilidad de enviar señales remotas a un centro de control del operador de red para que el manejo de las fallas sea eficiente y económico, y así mejorar la relación costo - beneficio ya que disminuye el tiempo de restable- cimiento del servicio y, por lo tanto, los costos de las fallas, aumentando la confiabilidad del sistema. La frecuencia de operación de estos equipos en condiciones normales es de $50 / 60 \mathrm{~Hz}$ dependiendo de la frecuencia del sistema donde se instale [10].

\section{Analizadores de redes}

Los analizadores de redes comúnmente utilizados cumplen con las normas internacionales IEEE 519 e IEC61999-1-4 Clase 1. Estos equipos permiten analizar sistemas de potencia capturando el valor eficaz de las señales de tensión y corriente cada medio ciclo, permiten medir tensión, corriente y potencia, y por lo tanto, calcular cualquier parámetro de calidad de potencia. Adicionalmente, por medio de software tiene la posibilidad de realizar análisis estadísticos y tendencias con el fin de determinar los problemas que se presentan en los sistemas, además de las pérdidas económicas. Estos equipos funcionan en condiciones normales para señales con frecuencia de hasta $3 \mathrm{kHz}$ [11].

Este tipo de analizadores funcionan por medio de sondas individuales de tensión y corriente para cada fase, neutro y tierra; esto hace que sea fácilmente configurable para diferentes topologías del sistema. Asimismo, algunos analizadores de redes cuentan con conexión Ethernet para la adquisición de los datos registrados en tiempo, y ranuras para memorias extraíbles que permiten almacenar gran cantidad de información y analizar los sistemas en amplios periodos de tiempo [12].

Los equipos más avanzados para analizar fenómenos de calidad de potencia pueden ser integrados a sistemas de control y gestión remotos y forman parte integral del control de las subestaciones y las redes en general, por lo cual facilitan el control del sistema de potencia haciéndolo más eficiente. Debido a esto, los operadores de red y las entidades gubernamentales involucradas en el control del sistema eléctrico de potencia tienden a implementar este tipo de sistemas en los nodos críticos del sistema, tales como fronteras comerciales, nodos de subestaciones y nodos en los cuales se presentan fallas regularmente.

Los mecanismos de registro mencionados anteriormente aplican para la medición de los fenómenos más comunes que afectan la calidad de potencia eléctrica para todos los niveles de tensión, sin embargo, para algunas aplicaciones estos dispositivos pueden ser costosos y difíciles de implementar ya que se requieren en diferentes nodos del sistema para obtener información confiable y lograr así la identificación del punto de falla o la dirección del cambio de la onda siguiendo su atenuación por la red.

Estos dispositivos tienen una frecuencia de muestreo de $100 \mathrm{kHz}$ para un nivel de tensión máximo de $6 \mathrm{kV} \mathrm{rms}$. Debido a estas características no es posible registrar sobretensiones transitorias en redes de media tensión, en especial las sobretensiones transito- 
rias de frente muy rápido, ya que tienen frecuencias de oscilaciones de hasta $300 \mathrm{kHz}$ [12]. Lo anterior ocasiona que los costos de los estudios de calidad de potencia para las redes de media tensión aumenten considerablemente, por lo tanto, se hace necesario desarrollar equipos para el registro de sobretensiones que sean económicos y de fácil implementación.

\section{Metodología}

Tras revisar los diferentes mecanismos para la medición de factores que afectan la calidad de potencia y demostrar que sus costos los hacen poco accesibles, se busca desarrollar una metodología que permita efectuar el registro de parámetros relevantes de las sobretensiones transitorias, y de esta manera, evaluar de forma sencilla y rápida las posibles causas que las ocasionan.

De esta forma, teniendo en cuenta que los equipos comúnmente utilizados para medir y registrar fenómenos de calidad de potencia eléctrica tienen mecanismos de medición invasivos, y por lo tanto, complejos en su instalación e implementación, y que para poder obtener la información completa y confiable de la red bajo estudio se deben instalar equipos en diferentes puntos, éstos se convierten en opciones nada rentables económicamente debido al costo individual de cada equipo. A partir de lo expuesto anteriormente, se propone desarrollar una metodología alternativa enfocada en registrar parámetros importantes de las sobretensiones transitorias en redes de media tensión, teniendo como base un prototipo que hace uso de un sensor capacitivo de campo eléctrico no invasivo, económico y de instalación sencilla. Con este prototipo se van a registrar los siguientes parámetros de las sobretensiones transitorias dentro de unos rangos específicos:

- Valor pico

- Duración

- Frecuencia de oscilación (para sobretensiones de frente muy rápido)

En la Fig. 1 se ilustran las etapas a tener en cuenta para el funcionamiento del prototipo y cómo se incorpora entre la red con las sobretensiones transitorias que la afectan y los datos y resultados que requiere el usuario para identificar la causa de la perturbación y una posible solución.

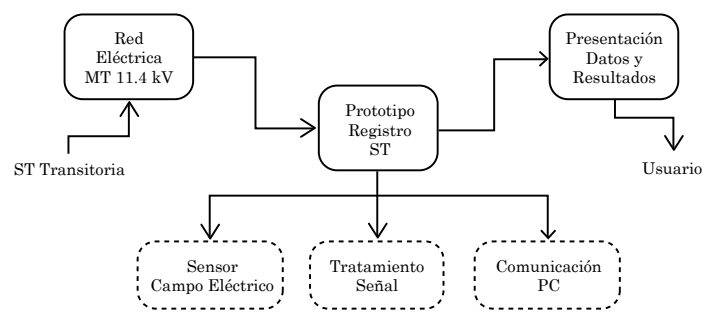

Fig. 1. Esquema general del prototipo para registrar parámetros de sobretensiones en redes de media tensión. Fuente: Autor.

\section{A. Sensor de campo eléctrico}

El sensor de campo eléctrico basa su funcionamiento en el principio de operación de un divisor capacitivo, es decir, utilizando el sensor como una capacitancia conocida se genera una capacitancia parásita entre la línea y el sensor. Por medio de este conjunto se garantiza que el nivel de tensión visto desde el sensor sea de algunos voltios [13]. El sensor a implementar está conformado por dos placas circulares paralelas con una separación y un grosor específicos, aspectos que junto con el radio de las placas definen el valor de la capacitancia del sensor como en (1):

$$
C_{\text {sensor }}=\frac{\pi * r^{2} * \varepsilon_{0}}{d}
$$

Donde:

$r \rightarrow$ Radio de las placas circulares [m]

$\varepsilon_{0}=8.854 * 10^{-12} \rightarrow$ Permitividad dieléctrica en el vacío $[\mathrm{F} / \mathrm{m}]$

$d \rightarrow$ Distancia de separación entre las placas circulares paralelas [m]

Para deducir un valor aproximado de la capacitancia generada entre la línea y el sensor se debe simular la totalidad del sistema, de esta manera se estimarán las tensiones producidas en el sensor a partir del divisor capacitivo, lo cual reduce las tensiones a unos cuantos voltios, niveles más sencillos de trabajar por medio de la electrónica básica. El diseño completo del sensor de campo eléctrico se presenta en [14].

El sensor recibe la señal de la sobretensión proveniente del sistema eléctrico, señal que está dada en voltios conservando una relación directamente proporcional de 1000 a 1 . Esta señal debe ser tratada para adquirir únicamente los parámetros de interés para la adecuada clasificación de la sobretensión transitoria según su posible causa, lo anterior manteniendo la siguiente etapa dentro de las condiciones de economía y efectividad frente a los equipos convencionales.

\section{B. Tratamiento de la señal}

En esta fase se realiza el acondicionamiento de la señal adquirida por el sensor y de la cual se deben obtener ciertos parámetros. Lo anterior se obtiene por medio de la utilización de dispositivos electrónicos, procurando siempre mantener la simplicidad del mecanismo en términos de cantidad de elementos electrónicos a utilizar para reducir al máximo los factores externos que pueden llegar a afectar y/o distorsionar la señal original y, por ende, desviar el resultado que se obtenga. Al elaborar esta fase por etapas se garantiza su modularidad, la verificación de errores y la posibilidad de realizar mejoras a futuro. A continuación, en la Fig. 2 se muestran las partes que conforman la fase de tratamiento de la señal. 


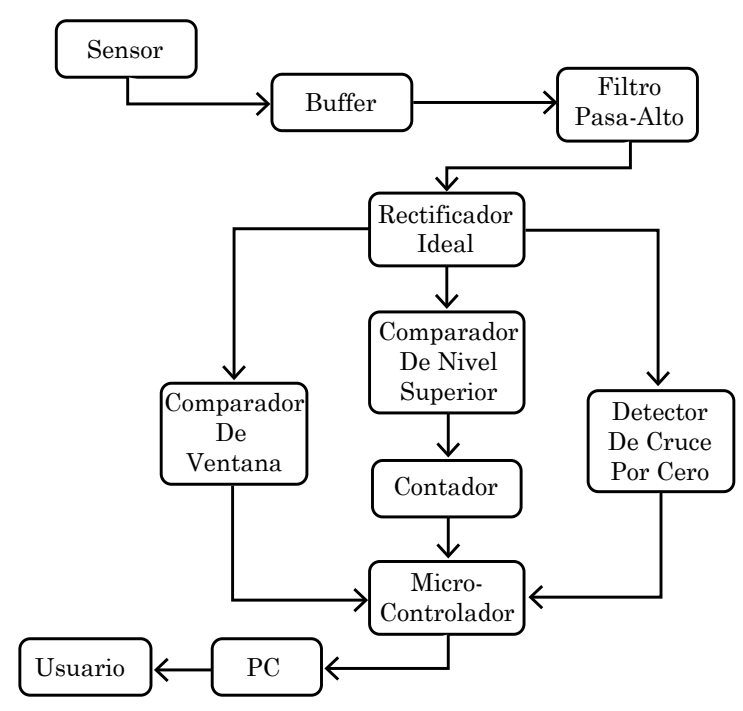

Fig. 2. Diagrama de bloques del tratamiento electrónico de la señal adquirida. Fuente: Autor.

Como primer elemento entre la señal y esta fase se emplea un buffer, el cual se utiliza para el acople de impedancias en el sistema. Posteriormente, se utiliza un filtro pasa-altos para eliminar las frecuencias menores a $1 \mathrm{kHz}$, ya que las frecuencias de interés se encuentran por encima de los $30 \mathrm{kHz}$ [1]. La siguiente etapa es un rectificador que permite discriminar las componentes positivas y negativas, las sobretensiones transitorias positivas son con frecuencia más críticas para un sistema eléctrico que las sobretensiones transitorias negativas [15] y [16], por tal razón las componentes negativas no serán evaluadas, sin embargo, en la configuración del rectificador implementado se puede recuperar la parte de la componente negativa de ser necesaria para la realización de un estudio más detallado. Adicionalmente, este elemento permite aislar la sobretensión dejando únicamente esta perturbación, mientras que el resto de la señal senoidal y el ruido propio de la red están en ceros.

Luego se presentan tres etapas adicionales las cuales deben disponerse en paralelo para que la señal de entrada a éstas sea la misma en el mismo momento: la primera de estas etapas es una configuración de comparadores de ventana, los cuales permiten identificar dentro de qué rango determinado se encuentra el valor pico de la sobretensión transitoria; la segunda etapa es otra configuración de comparador, pero esta vez de nivel superior, al cual se le establece un nivel de comparación cercano a cero para que al pasar dicho umbral active un contador y de esta manera se pueda establecer el rango de tiempo para obtener la duración del evento; la tercera etapa consiste en un detector de cruce por cero con el cual se podrá verificar si la señal obtenida es oscilatoria.
Después de obtener estas diferentes salidas, todas de naturaleza análoga, se prosigue a direccionar esas salidas a los puertos del microcontrolador previamente programado, y de esta manera, utilizar las virtudes de este elemento para realizar la comunicación entre la fase electrónica y el PC, convirtiendo la información adquirida en datos admisibles para el puerto USB del computador. En la Tabla II se muestran las categorías que se establecen a partir de los parámetros de evaluación.

Tabla II. Categorías para Clasificación de Sobretensiones Transitorias.

\begin{tabular}{|c|c|c|c|}
\hline \multirow{2}{*}{ Categoría } & \multicolumn{3}{|c|}{ Parámetro } \\
\cline { 2 - 4 } & $\begin{array}{c}\text { Valor } \\
\text { pico (p.u.) }\end{array}$ & $\begin{array}{c}\text { Frecuencia de } \\
\text { oscilación (kHz) }\end{array}$ & $\begin{array}{c}\text { Duración } \\
\text { (s) }\end{array}$ \\
\hline I & N/I & N/I & N/I \\
\hline II & $1.5-4$ & NA & $\leq 20 \mathrm{~ms}$ \\
\hline III & $4-6$ & NA & $\leq 300 \mathrm{us}$ \\
\hline IV & $6-10$ & $30-300$ & $\leq 3 \mathrm{~ms}$ \\
\hline
\end{tabular}

Fuente: Autor.

- Categoría I: El equipo no registra información.

- Categoría II: Sobretensión ocasionada por maniobras.

- Categoría III: Sobretensión ocasionada por descargas atmosféricas.

- Categoría IV: Sobretensión ocasionada por descargas atmosféricas inducidas.

La evaluación de los parámetros se hace iniciando por el valor pico, continuando con la duración del fenómeno y, por último, la frecuencia de oscilación. De esta forma se pueden descartar las categorías a las que no pertenece el fenómeno. Además, es importante evaluar mínimo dos parámetros para establecer una mayor certeza en el resultado obtenido.

En el siguiente ejemplo se clarifica el método de utilización de la Tabla II para la categorización de las sobretensiones transitorias.

\section{- Datos obtenidos por el prototipo:}

Valor pico (p.u.) = se registra información al límite de las categorías III y IV.

Duración $(\mathrm{s})$ = se registra información que puede pertenecer a las categorías III y IV.

Frecuencia de oscilación $(\mathrm{kHz})=$ se registra información.

\section{- Evaluación de información:}

El valor pico obtenido se encuentra en el límite de las categorías III y IV, por lo cual se debe evaluar un segundo parámetro. Al validar que la duración obtenida vuelve a encontrarse contenida en las categorías III y IV, se hace necesaria la evaluación del último parámetro. Se presenta una frecuencia de os- 
cilación de $50 \mathrm{kHz}$, descartando así la categoría III puesto que ésta no presenta dicho parámetro, dejando como categoría resultante la IV (sobretensión ocasionada por descargas atmosféricas inducidas). De esta forma se descartan las categorías que no presentan datos en sus rangos establecidos. Aunque se dispone un orden para evaluar los datos en los parámetros, se puede optar por revisar el más relevante, por ejemplo, si el evento es oscilante se adjudicara el fenómeno directamente a la categoría IV.

Adicionalmente, se elabora una interfaz usuario - máquina en LabView que permite mostrar de manera sencilla y agradable al usuario los resultados obtenidos por el prototipo de registro de sobretensiones transitorias.

\section{Resultados}

Puesto que la metodología de registro tiene como objetivo categorizar la sobretensión transitoria mas no reproducir la señal ni presentar valores exactos de los parámetros de interés de la onda, se establece que este prototipo es un elemento de calidad estadística.

La identificación de las posibles causas que generan la sobretensión transitoria en el sistema es suficiente para orientar los esfuerzos a la mitigación y protección de la red sin necesidad de poseer los valores exactos de los eventos, puesto que la realización de estudios previos en la calidad de potencia ya permiten conocer las características específicas de estos disturbios, por lo cual solamente se requiere ubicar los puntos más susceptibles y frágiles para adoptar cuidados específicos frente a sus causas y reducir así sus efectos.

Para la implementación del prototipo propuesto no es necesaria la realización de ninguna maniobra de corte del servicio eléctrico, ya que no requiere de una conexión física con la línea. Esta es otra de las razones por las cuales su ubicación en diferentes puntos de la red es sencilla y rápida. Adicionalmente, la posible pérdida de un ejemplar afecta de forma mínima el aspecto económico del estudio ya que la suma del valor comercial de sus elementos constructivos en conjunto no superara el valor de ningún equipo de medición de calidad de potencia eléctrica convencional.

\section{Conclusiones}

El estudio de los fenómenos de calidad de potencia eléctrica cobra gran importancia puesto que tienen efectos negativos tanto técnicos como económicos sobre las instalaciones afectadas y los usuarios conectados a ellas. Sin embargo, las diferentes metodologías implementadas para la medición de parámetros de calidad de potencia son invasivas y requieren de sondas en cada uno de los puntos o nodos donde se va a realizar la medición, lo cual no resulta conveniente para las diferentes topologías de red utilizadas.

La metodología propuesta busca facilitar el desarrollo de estrategias para la mitigación de los efectos negativos de las sobretensiones transitorias en redes de media tensión, específicamente la red de $11.4 \mathrm{kV}$.

El prototipo que forma parte de la metodología planteada se puede instalar fácilmente en diferentes nodos del sistema para la realización de un estudio de calidad de potencia debido a su bajo costo y su mecanismo de registro no invasivo. Además, está orientado al desarrollo de estudios de naturaleza estadística mas no determinística, lo anterior obedece al uso de rangos de datos y no de valores exactos.

La modularidad que se plantea para el prototipo desde el sensor, pasando por la fase de adquisición y tratamiento de datos hasta la interfaz usuario máquina permitirá realizar mejoras a futuro, tales como: adaptación a diferentes topologías de red, adecuación para otros niveles de tensión, entre otras. De igual manera, al elaborar la fase electrónica por etapas se puede realizar verificación de errores paso a paso para obtener una mayor confiabilidad en los resultados.

\section{REFERENCIAS}

[1] High Voltage Engineering Sectional Committee, IS/IEC 60071-1. Insulation Co-ordination, Part 1: Definitions, Principles and Rules. India, 2006.

[2] M. Lanphier, P. K. Sen y J. P. Nelson, "An Update on Surge Protection of Medium Voltage Motors: A Comparison of the Standards and Applications," in 2007 4th European Conference on Electrical and Instrumentation Applications in the Petroleum \& Chemical Industry, 2007, pp. 1-8.

[3] M. A. Omidiora y M. Lehtonen, "Universities Power Engineering Conference (UPEC), 2009 Proceedings of the 44th International," in Universities Power Engineering Conference (UPEC), 2009 Proceedings of the 44th International, 2009, pp. 1-5.

[4] "IEEE Std. C62.21-2003/Cor 1-2008 (Corrigendum to IEEE Std C62.21-2003)," in IEEE Std. C62.21-2003/Cor 1-2008 (Corrigendum to IEEE Std C62.21-2003), 2008, pp. 1-12.

[5] S. Wang, C. Sun, L. Zhang, L. Du y S. Xi, "Identifying the Internal and the External Overvoltage of Distribution Networks Based on Fisher Discriminate Method," in 2006 International Conference on Power System Technology, 2006, pp. 1-4.

[6] J. A. Martínez Velazco y F. Castro Aranda, Análisis de sobretensiones de origen atmosférico en líneas aéreas de transporte, Catalunya: Universitat politécnica de Catalunya, 2002.

[7] M. Vassileva, M. Yordanova, Y. Rangelov y N. Nikolaev, "Simulation of the process of arising and limitation of lightning surges in medium voltage power grids," in 2013 12th International Conference on Environment and Electrical Engineering, 2013, pp. 180-185.

[8] P. N. Mikropoulos, T. E. Tsovilis, Z. Politis y A. G. Kagiannas, "Evaluation of fast-front overvoltages arising at a $20 / 0.4 \mathrm{kV}$ distribution transformer," in 7th Mediterranean Conference and Exhibition on Power Generation, Transmission, Distribution and Energy Conversion (MedPower 2010), 2010, pp. 191-191. 


\section{METODOLOGÍA PARA LA MEDICIÓN DE PARÁMETROS DE SOBRETENSIONES TRANSITORIAS EN REDES DE DISTRIBUCIÓN DE $11.4 \mathrm{KV}$}

[9] Fluke, "Registrador trifásico de calidad eléctrica Fluke 1750." [En línea]. Disponible en: http://www.fluke.com/ fluke/coes/Medidores-de-Calidad-de-la-Energia-Electrica/Logging-Power-Meters/Fluke-1750.htm?PID=56030 [Consultado en ago. 9, 2014]

[10] V. A. Gómez, R. A. Peña y C. Hernández, "Identificación y localización de fallas en sistemas de distribución, utilizando medidores de calidad del servicio de energía eléctrica," Rev. Científica, no. 14, pp. 177-184, jul. 2012.

[11] Fluke, "Búsqueda de manuales Fluke." [En línea]. Disponible en: http://assets.fluke.com/manuals/1730 umspa0000.pdf [Consultado en ago. 8, 2014].

[12] Fluke, "Logging power Meters." [En línea]. Disponible en: http://www.fluke.com/fluke/coes/Medidores-de-Calidad-de-la-Energia-Electrica/Logging-Power-Meters/ Fluke-435-Series-II.htm?PID=73939 [Consultado en ago. 9, 2014].
[13] C. F. Ramírez, Subestaciones de alta tensión y extra alta tensión, 2nd ed. Medellín, 2003.

[14] D. Warne y A. Haddad, Advances in High Voltage Engineering. Londres: IET, 2004.

[15] J. F. Perkins, "Evaluation of Switching Surge Overvoltages on Medium Voltage Power Systems," IEEE Trans. Power Appar. Syst., vol. PAS-101, no. 6, pp. 1727-1734, jun. 1982.

[16] B. Eliert y M. Runar, "Efficient Outage Management using Fault Passage Indicators," in International Conference on T\&D Asset management for electric utilities, 2008. 